\title{
Exotic charmonium and light-by-light scattering in heavy ion collisions
}

\author{
V.P. Gonçalves, B.D. Moreira \\ Instituto de Física e Matemática, Universidade Federal de Pelotas \\ Caixa Postal 354, CEP 96010-900, Pelotas, RS, Brazil \\ barros@ufpel.edu.br, bduartesm@gmail.com
}

\section{R. Molina}

Instituto de Física, Universidade de São Paulo, Rua do Matão, 1371 Butantã, C.P. 05508-090

São Paulo, Brazil

ramopedif.usp.br

\section{F. S. Navarra*}

Instituto de Física, Universidade de São Paulo, Rua do Matão, 1371 Butantã, C.P. 05508-090

São Paulo, Brazil

Institut de Physique Théorique, Université Paris Saclay, CEA, CNRS, F-91191,

Gif-sur-Yvette, France

navarradif.usp.br

The internal structure of exotic charmonium $(\mathrm{R})$ states is still a subject of debate. Some of the popular pictures used to describe them are tetraquark, meson molecule or mixtures of those with charmonium. One way to shed light into the nature of these states is to study their radiative decays. We expect that their decay widths into two photons will vary from one configuration to another. The direct experimental measurement of these widths in the reaction $R \rightarrow \gamma \gamma$ is difficult. However, they manifest themselves also indirectly in the production, through photon-photon fusion, of the resonance $R$ in ultraperipheral nucleus-nucleus collisions (UPHIC), where the production cross section is directly proportional to the decay width $\Gamma(R \rightarrow \gamma \gamma)$. Once produced, the resonance $R$ will decay mostly in hadronic channels but it may also decay into a photon pair. In this case, the cross section will be proportional to the squared $2 \gamma$ decay width, being even more sensitive to the internal structure of the resonance. In this note we review the predictions for exotic charmonium production in UPHIC and present, for the first time, estimates of the production cross section of exotic states which decay into two photons.

XVII International Conference on Hadron Spectroscopy and Structure - Hadron 2017 25-29 September, 2017

University of Salamanca, Salamanca, Spain

\footnotetext{
*Speaker.
} 


\section{Introduction}

The existence of many charmonium exotic states (here called simply $R$ ) is well established [1]. In the near future we hope to understand better their properties. In the literature [1] the most frequently used models to describe the $R$ states are the tetraquark and the meson molecule model (a mixture of these states with charmonium is also possible). One way to distinguish one model from the other is to explore their different spatial configurations: tetraquarks are compact $(r \simeq 0.2-0.5 \mathrm{fm})$ whereas molecules are large $(r \simeq 3.0-6.0 \mathrm{fm})$ objects. Thus, we expect that, due to this difference, their decay widths into two photons will be significantly different. The direct experimental measurement of these widths, i.e. $R \rightarrow \gamma \gamma$, is difficult. However, as we will argue below, they manifest themselves also indirectly in the production, through photon-photon fusion, of the resonance $R$ in ultraperipheral nucleus-nucleus collisions, where the production cross section is directly proportional to the decay width $\Gamma(R \rightarrow \gamma \gamma)$.

There are many possible experimental ways to study the new R states. Among them, heavy ion collisions (HIC's) are an interesting option because of the large number of heavy quarks produced in these collisions [2]. HIC's can be central, non-central and ultraperipheral (UPHIC), where the impact parameter is very large and the interaction between the projectile nucleus $A$ and the target nucleus $B$ is purely electromagnectic [3]. In this case, the state $R$ can be formed through $\gamma \gamma$ fusion: $\gamma \gamma \rightarrow R$. Recently, we studied the production of exotic charmonium in UPHIC's [4] and concluded that the exclusive production of resonances $\mathrm{R}$ in the process $A B \rightarrow A B R$ has a relatively large cross section, as it can be seen in Table I. After being produced, the resonance decays and the decay products may be detected. The hadronic decays are favored and we will discuss them in more detail elsewhere. In this note we focus on the two photon decay channel. The reaction is then: $A+B \rightarrow A+B+\gamma+\gamma$. UPHIC reactions with the exclusive production of two photons (also called light-by-light scattering) started to be measured very recently by the ATLAS collaboration [5]. Indeed, in this paper, the fiducial cross section of the process $P b+P b(\gamma \gamma) \rightarrow P b+P b \gamma \gamma$, for photon transverse energy larger than $3 \mathrm{GeV}$, photon absolute pseudorapidity $|\eta|<2.4$ and diphoton invariant mass greater than $6 \mathrm{GeV}$, was found to be $70 \pm 24$ (stat.) \pm 17 (syst.) nb. In the future, with better statistics, it may be possible to determine the two-photon invariant mass spectrum in these collisions. Looking at the structures in this spectrum it may be possible to identify resonances. The process $\gamma+\gamma \rightarrow \gamma+\gamma$ receives all kinds of contributions, as discussed in Ref. [6]. However, these different contributions can be, to some extent, separated in a careful analysis of the two-photon invariant mass distribution $M_{\gamma \gamma}$. Moreover, as it has been shown in recent calculations [7], in the case where scalar resonances are exchanged, the s-channel produces a pronounced peak. In this first estimate we will thus consider only s-channel processes, where the state $R$ is produced and decays into two photons. We will not include other diagrams.

\section{The reaction $\mathrm{PbPb} \rightarrow \mathrm{PbPbR}$}

In ultraperipheral processes the impact parameter is bigger than the sum of the radii of the colliding particles and hence the projetile and the target do not overlap. The interaction is purely electromagnetic and resonance production results from photon-photon fusion. The cross section is 


\begin{tabular}{|c|c|c|c|c|c|c|c|c|}
\hline State & \multirow{2}{*}{ Mass } & $\Gamma_{\gamma \gamma}^{\text {theor }}(\mathrm{keV})$ & \multicolumn{3}{|c|}{$\sigma_{b_{\min }}(\mu \mathrm{b})$} & \multicolumn{3}{c|}{$\sigma_{F}(\mu \mathrm{b})$} \\
\cline { 4 - 9 } & & & $2.76 \mathrm{TeV}$ & $5.5 \mathrm{TeV}$ & $39 \mathrm{TeV}$ & $2.76 \mathrm{TeV}$ & $5.5 \mathrm{TeV}$ & $39 \mathrm{TeV}$ \\
\hline \hline $\mathrm{X}(3940), 0^{++}$ & 3943 & 0.33 & 4.2 & 8.2 & 31.6 & 6.5 & 11.8 & 40.9 \\
$\mathrm{X}(3940), 2^{++}$ & 3943 & 0.27 & 17.2 & 33.6 & 129.2 & 26.5 & 48.4 & 167.4 \\
$\mathrm{X}(4140), 0^{++}$ & 4143 & 0.63 & 6.5 & 12.9 & 51.2 & 10.2 & 18.7 & 65.7 \\
$\mathrm{X}(4140), 2^{++}$ & 4143 & 0.50 & 26.0 & 51.2 & 201.0 & 40.3 & 74.3 & 260.6 \\
$\mathrm{Z}(3930), 2^{++}$ & 3922 & 0.04 & 5.4 & 10.5 & 40.9 & 8.3 & 15.2 & 52.4 \\
$\mathrm{X}(4160), 2^{++}$ & 4169 & 0.18 & 18.4 & 36.4 & 144.2 & 28.6 & 52.7 & 185.3 \\
$\mathrm{Y}_{p}(3912), 2^{++}$ & 3919 & 0.38 & 50.5 & 98.6 & 382.4 & 77.9 & 142.2 & 490.1 \\
$\mathrm{X}(3915), 0^{++}$ & 3919 & 0.20 & 2.6 & 5.1 & 19.8 & 4.0 & 7.3 & 25.3 \\
\hline
\end{tabular}

Table 1: Cross sections for exotic meson production in $P b-P b$ collisions. $\sigma_{b_{\min }}$ and $\sigma_{F}$ refer to different choices for the nuclear form factor, as explained in Ref. [4]. The theoretical decay width $\Gamma_{\gamma \gamma}^{\text {theor }}$ was taken from Refs. [8, 9].

given by [4]:

$$
\sigma(A B \rightarrow A B R)=\int \hat{\sigma}(\gamma \gamma \rightarrow R) N\left(\omega_{1}, \mathbf{b}_{1}\right) N\left(\omega_{2}, \mathbf{b}_{2}\right) \mathrm{d}^{2} \mathbf{b}_{1} \mathrm{~d}^{2} \mathbf{b}_{2} \mathrm{~d} \omega_{1} \mathrm{~d} \omega_{2},
$$

where $N\left(\omega_{i}, b_{i}\right)$ is the equivalent photon spectrum generated by the nucleus $i$ and $\sigma(\gamma \gamma \rightarrow R)$ is the cross section for the production of a state $R$ from two real photons with energies $\omega_{1}$ and $\omega_{2}$. In Eq.(2.1), $\mathbf{b}_{1}$ and $\mathbf{b}_{2}$ are the distances from the center of nucleus $A$ and $B$ and they satisfy the UPHIC condition $\left|\mathbf{b}_{1}-\mathbf{b}_{2}\right|>R_{A}+R_{B}$, where $R_{A}$ and $R_{B}$ are the radius of nucleus $\mathrm{A}$ and $\mathrm{B}$ respectively. The $\gamma \gamma$ fusion cross section is given by the Low formula:

$$
\sigma_{\gamma \gamma \rightarrow R}\left(\omega_{1}, \omega_{2}\right)=8 \pi^{2}(2 J+1) \frac{\Gamma_{R \rightarrow \gamma \gamma}}{M_{R}} \delta\left(4 \omega_{1} \omega_{2}-M_{R}^{2}\right),
$$

In order to estimate the cross section of the process $A+B \rightarrow A+B+\gamma+\gamma$, which occurs via $\gamma+\gamma \rightarrow R \rightarrow \gamma+\gamma$, we just have to multiply the cross section (2.1) by the probability of the resonance to decay into two photons, i.e. :

$$
\sigma(A B \rightarrow A B \gamma \gamma)=\sigma(A B \rightarrow A B R) \cdot \frac{\Gamma_{R \rightarrow \gamma \gamma}}{\Gamma_{R}}
$$

where $\Gamma_{R}$ is the total decay width of the state R. Inserting (2.2) into (2.1) and this latter into (2.3) we observe that the cross section above is proportional to the square of the decay width $\Gamma_{R \rightarrow \gamma \gamma}$, which is not very well known and strongly depends on the nature (and spatial configuration) of the resonance $\mathrm{R}$.

For an S-wave non-relativistic two-body system $\mathrm{R}$ in a state described by a wave function $\psi(r)$ the width for annihilation into $\gamma \gamma$ is given by

$$
\Gamma(R \rightarrow \gamma \gamma)=\frac{2 \pi \alpha^{2}}{M_{R}^{2}}|\psi(0)|^{2}
$$

From this formula we can see that the decay width is proportional to the wave function at the origin. The larger it is, the easier the components can meet and annihilate into photons. This might 


\begin{tabular}{|c|c|c|c|c|c|}
\hline State & $\Gamma_{R}(\mathrm{MeV})$ & $\Gamma(R \rightarrow \gamma \gamma)(\mathrm{keV})$ & \multicolumn{3}{|c|}{$\sigma_{P b P b P b P b \gamma \gamma}(\mathrm{pb})$} \\
\cline { 4 - 6 } & & & $2.76 \mathrm{TeV}$ & $5.02 \mathrm{TeV}$ & $39 \mathrm{TeV}$ \\
\hline \hline $\mathrm{X}(3940)$ & 37 & 0.33 & 57.7 & 98.1 & 369.0 \\
\hline $\mathrm{X}(4140)$ & 19 & 0.63 & 334.9 & 573.8 & 2193.5 \\
\hline $\mathrm{X}(3915)$ & 20 & 0.20 & 40.3 & 68.3 & 255.5 \\
\hline
\end{tabular}

Table 2: $\mathrm{PbPb} \rightarrow \mathrm{PbPb} \gamma \gamma$ cross section.

be more difficult for a charm meson molecule, since in this case, the annihilation occurs through the exchange of a heavy meson (typically a $D$ or a $D^{*}$ ), which range is of the order of $1 / m_{D} \simeq 0.2$ $\mathrm{fm}$, while the typical separation between the mesons in the molecule is $r \simeq 3-6 \mathrm{fm}$. This mismatch between the scales leads to a suppression of the radiative decays of molecular states. In contrast, for tetraquarks, the state is compact, the wave function at the origin is large and consequently the decay width is large.

In the molecular picture, several calculations were done in the past. In Ref. [8] the Weinberg's compositeness condition together with Heavy Hadron Chiral Perturbation Theory was used to evaluate the radiative decay width of the X(3940), which was found to be $\Gamma(R \rightarrow \gamma \gamma)=0.33 \pm 0.01$ keV. In Ref. [9] the exotic states were dynamically generated in the Hidden Gauge Formalism (HGF) with values of the radiative decay widths $\Gamma(R \rightarrow \gamma \gamma)=0.04-0.08 \mathrm{keV}$. These two results are quite different and clearly further investigation needs to be done. In the tetraquark approach, using QCD sum rules, the authors of Ref. [10] found $\Gamma(R \rightarrow \gamma \gamma)=1.6 \pm 1.3 \mathrm{keV}$. The errors are large, but the numbers are suggestive. Taking the central values, we see that the tetraquark width is about one order of magnitude larger than the molecule one, and so is the production cross section in UPHIC.

\section{Results and discussion}

In Fig. 1 we show the cross section of the reaction $P b P b \rightarrow P b P b \gamma \gamma$ and also the rapidity distribution of the $\gamma \gamma$ pair. For the sake of completeness we also show in Table II the values of the cross sections at some relevant energies. The comparison of Table I with Table II (and also of Fig. 1 with the analogous figure published in Ref. [4]) shows that, because of the extra factor $\Gamma(R \rightarrow \gamma \gamma)$ on the rhs of Eq. (2.3), the difference between the $X(3940)$ and $X(4140)$ cross sections grows from a factor $\simeq 1.55$ to a factor $\simeq 5.8$. This indicates that the analysis of this process (production plus decay into the two photon channel) will also allow for a better discrimination of the $\mathrm{R}$ states. A striking consequence of the above considerations, also due to the extra factor $\Gamma(R \rightarrow \gamma \gamma)$, is the fact that the cross section for producing a tetraquark becomes two orders of magnitude larger than the cross section for producing a meson molecule. Finally, the absolute numbers shown in Table II suggest that these processes, albeit rare, might be observed, specially in the case of tetraquarks. This encourages us to study this subject in more detail. 

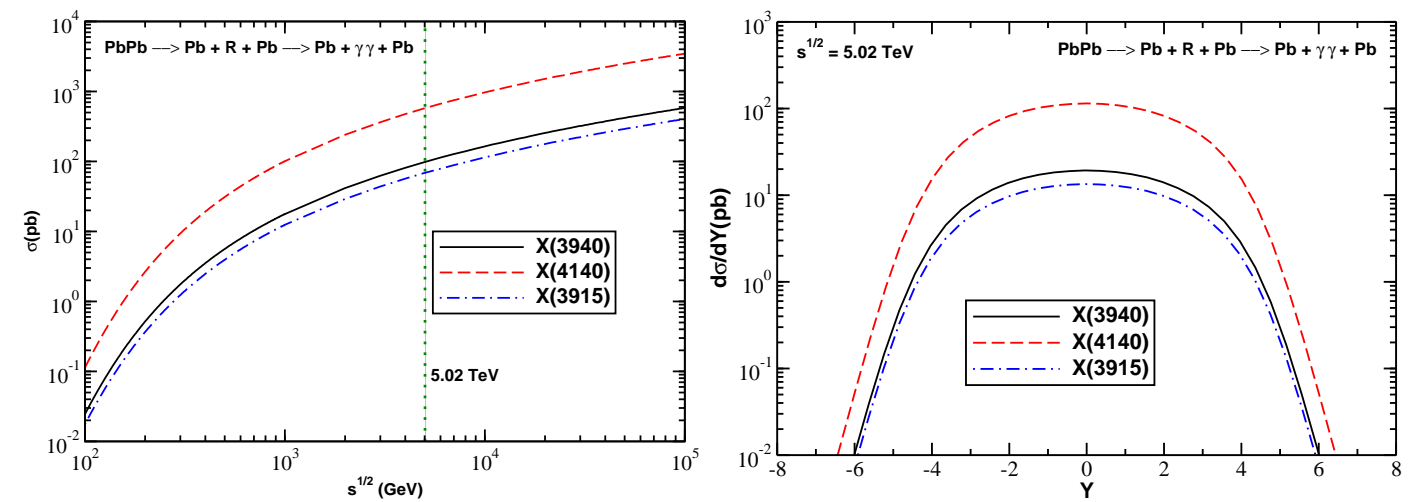

Figure 1: $\mathrm{PbPb} \rightarrow \mathrm{PbPb} \gamma \gamma$ cross section (left) and rapidity distribution of the $\gamma \gamma$ pair (right).

\section{References}

[1] A. Hosaka, T. Iijima, K. Miyabayashi, Y. Sakai and S. Yasui, PTEP 2016, 062 C01 (2016); F. K. Guo, C. Hanhart, U. G. Meissner, Q. Wang, Q. Zhao and B. S. Zou, arXiv:1705.00141; A. Esposito,

A. L. Guerrieri, F. Piccinini, A. Pilloni, A. D. Polosa, Int. J. Mod. Phys. A 30, 1530002 (2014); M. Nielsen, F. S. Navarra, Mod. Phys. Lett. A 29, 1430005 (2014); R. Molina and E. Oset, Phys. Rev. D 80, 114013 (2009); T. Branz, T. Gutsche and V. E. Lyubovitskij, Phys. Rev. D 80, 054019 (2009);

R. D. Matheus, F. S. Navarra, M. Nielsen and C. M. Zanetti, Phys. Rev. D 80, 056002 (2009).

[2] S. Cho et al. (ExHIC Collaboration), Prog. Part. Nucl. Phys. 95, 279 (2017).

[3] C. A. Bertulani, S. R. Klein and J. Nystrand, Ann. Rev. Nucl. Part. Sci. 55, 271 (2005).

[4] B. D. Moreira, C. A. Bertulani, V. P. Goncalves and F. S. Navarra, Phys. Rev. D 94, 094024 (2016).

[5] A. Aaboud (ATLAS Collaboration), Nature Phys. 13, 852 (2017).

[6] A. Szczurek and M. Klusek-Gawenda, arXiv:1710.10868; M. Klusek-Gawenda, P. Lebiedowicz and A. Szczurek, Phys. Rev. C 93, 044907 (2016).

[7] P. Lebiedowicz and A. Szczurek, Phys. Lett. B 772, 330 (2017).

[8] T. Branz, T. Gutsche and V. E. Lyubovitskij, Phys. Rev. D 80, 054019 (2009).

[9] T. Branz, R. Molina and E. Oset, Phys. Rev. D 83, 114015 (2011).

[10] R. M. Albuquerque, J. M. Dias, M. Nielsen and C. M. Zanetti, Phys. Rev. D 89, 076007 (2014). 